\title{
The effect and long-term outcome of Partial Anterior Myometrial Resection and Reconstruction under tourniquet and/or prophylactic abdominal aorta balloon occlusion on the patients with placenta percreta
}

\section{Huidan Zhao}

Zhengzhou University First Affiliated Hospital https://orcid.org/0000-0002-1583-8354

\section{Chen Chen}

Zhengzhou University First Affiliated Hospital

\section{Ya Tao}

Zhengzhou University First Affiliated Hospital

Zhuan Liu

Zhengzhou University First Affiliated Hospital

Cai Liu

Zhengzhou University First Affiliated Hospital

Xianlan Zhao ( $\nabla$ zxl12129@126.com )

https://orcid.org/0000-0002-0615-4487

\section{Research article}

Keywords: placenta accreta spectrum (PAS), placenta percreta, abdominal aorta, balloon occlusion

Posted Date: June 8th, 2020

DOl: https://doi.org/10.21203/rs.3.rs-31115/v1

License: (c) (i) This work is licensed under a Creative Commons Attribution 4.0 International License.

Read Full License 


\section{Abstract \\ Background}

Patients with placenta accreta spectrum (PAS) disorders often experience overwhelming hemorrhage during cesarean. Placenta percreta is the most serious subtype. In this paper, we propose a new procedure in order to control intraoperative bleeding. We aimed to evaluate the effect and long-term outcome of Partial Anterior Myometrial Resection and Reconstruction under tourniquet and/or prophylactic abdominal aorta balloon occlusion on patients with placenta percreta.

\section{Methods}

In a retrospective study, data from patients with placenta percreta who delivered by cesarean section between January 1, 2017 and December 31, 2019 were analyzed. Short-term and long-term outcomes were followed up by outpatient clinic and by phone. The quantity of estimated blood loss (EBL), operation time, urine tube time, hospital stay and short-term and long-term complications including fever, thrombosis, hematoma, breast feeding and menstruation problems, lower limb complaints, intrauterine adhesion and so on, were analyzed.

\section{Results}

For all cases, the mean EBL in the surgery was $1399 \pm 948 \mathrm{~mL}$, the mean operation time was $107 \pm$ 35 minutes, and the mean perioperative hospital stay was $9.6 \pm 5.0$ days. All the patients had a preserved uterus. Menstrual quantity decreased in 12 patients. Menstrual period prolonged in 11 cases. Uncomfortable abdomen symptoms happened in 9 cases after the surgery. Four cases got thrombosis and 3 got hematoma. Lower extremity discomfort was found in 6 patients.

\section{Conclusion}

Combined with prophylactic abdominal aorta balloon occlusion and/or tourniquet, Partial Anterior Myometrial Resection and Reconstruction is highly effective in reducing the intraoperative blood loss and hysterectomy in placenta percreta. But we should pay great attention to short-term and long-term complications, especially to the complications associated with aorta balloon occlusion.

\section{Background}

With the implementation of the 2-child policy in China, more and more women of advanced maternal age are choosing to have another child. Over the past decades, the continuous increase in the cesarean delivery rate has resulted in a substantial rise in women with prior cesarean delivery scars [1]. Also, the use of assisted reproductive technology has increased remarkably. All these risk factors can contribute to 
placenta accrete spectrum (PAS) disorders. Placenta accreta spectrum is associated with a very high risk of obstetric and pediatric complication,especially postpartum hemorrhage(PPH) which often leads to secondary complications including shock, coagulopathy, disseminated intravascular coagulation (DIC), multi-organ failure(MOF), and even death [1,2]. Meanwhile, placenta accrete spectrum is likely to induce uterine rupture, uterine hysterectomy $[$ preterm labor and neonatal respiratory distress syndrome. A variety of uterus compression sutures for the management of placenta previa accrete have been reported, each with varying advantages and complications [3-9]. According to the invasive depth of trophoblast, three subtypes have been differentiated: placenta accreta, placenta increta and placenta percreta[1]. The invasive villous tissue penetrates to or through the uterine serosa and, in some cases, even to the adjacent organs in the placenta percreta situation. Therefore, placenta percreta is the most serious subtype and easily causes severe maternal and fetal complications. In this paper, we propose a new procedure called Partial Anterior Myometrial Resection and Reconstruction (PAMRR), as a method for treating placenta percreta, mainly focusing on the bleeding of the anterior myometrium which is the most common and serious part of placenta invaded. We report the follow-up results of 142 cases, including long-term and short-term outcomes.

\section{Methods}

\section{Patients}

A retrospective study was conducted on 142 patients with placenta percreta at The First Affiliated Hospital of Zhengzhou University between January 2017 and December 2019. The patients returned to the outpatient clinic of the hospital about 42 days after the surgery. Follow-up was made by phone call three months, six months, one year and two years after the surgery. The inclusion criteria were fulfilled by patients suffering from placenta percreta confirmed by ultrasound, Magnetic Resonance Imaging (MRI) and surgical manifestations. The exclusion criteria were met by patients who were unsuitable or unnecessary for the procedure, including cases of serious abdominal adhesion, small area placenta percreta, severe obstetric complications and serious internal and surgical diseases, such as serious heart disease, liver disease, pre-eclampsia and so on. All operations were performed by the same surgeon, Xianlan Zhao, with several constant assistants. The whole progress was managed by our multidisciplinary team, including doctors expert in ultrasound, MRI, anesthesiology, invasive technology and obstetrics.

\section{Surgical Procedure}

Before the surgery, we performed an ultrasonic examination to record information about the placenta, including its localization, thickness, loss of the hypoechoic retroplacental zone and numerous large and irregular lacunae in the placenta. For most of the patients, we did an MRI, if necessary, to grasp more details, including abnormal uterine bulging, dark intraplacental bands on T2-weighted images, heterogeneous signal intensity with the placenta and disorganized placental vasculature. According to 
the evidence, we made an individual schedule for each patient, covering gestational week to terminate the pregnancy, the amount of prepared blood, the necessity of placing a prophylactic abdominal aorta balloon and so on. We performed the operation in the Digital subtraction angiography (DSA) room.

1. The primary incision was recut again, and the scar was removed from all the patients who had undergone at least one C-Section before. The majority were transverse incisions of the lower abdomen and the minority were longitudinal incisions. But our incision was about $3 \mathrm{~cm}$ longer than the previous one in order to expose the upper border of the placenta.

2. The uterine incision was performed transversely over the upper border of the placenta which is obvious after opening the abdomen. As we see in Figure A, there were engorged and tortuous vessels on the serosa surface corresponding to the area of the placenta increta beneath. The incision was pulled tightly by the surgeon and the first assistant to keep some tension to minimize bleeding.

3. After delivering the fetus, the uterus was exteriorized immediately by the first assistant whose left hand pulled the anterior wall and the right hand dragged the fundus in the abdominal cavity.

Afterwards, we checked whether adhesion existed or not between the uterus and abdominal wall. The adhesion should be separated.

4. The aortic balloon which had been placed below the kidney artery before cesarean was inflated with simultaneous delivery to occlude blood flow to the uterus. Meanwhile, we put a tourniquet as low as possible at the lower segment of the uterus to further block blood flow to the uterus. It was difficult to establish a tourniquet on the patients with serious adhesion in which the aortic balloon was crucial and irreplaceable. Both treatments significantly reduced the amount of bleeding.

5 . We pushed down the bladder and partially resected the anterior myometrium with the placenta unseparated, as we see in Figure B. All of our patients had placenta percreta covering a large area. The bladder was pushed down to horizontal line of internal cervix os or to the accreta area if the posterior wall of the bladder muscle layer was invaded. The anterior myometrial from the lower lip of the uterine incision to area $1 \mathrm{~cm}$ above the cervix was resected with a scalpel or scissor together with the bulk of implanted placenta. Thanks to the prophylactic abdominal aorta balloon and/or tourniquet, bleeding hardly ever occurred during this process.

6. A single continuous suture was made along the lower lip of the uterine incision, as we see in Figure C. Because the new-formed lower lip was one part of placenta deeply implanted area and full of tortuous vessels which can cause massive bleeding in a short time, this single continuous suture could minimize the bleeding significantly. Other hemostatic methods were conducted on the placental bed to prevent bleeding, including the area around the internal cervix os and the area where the placenta could not be excised. Figure-of-eight suture was the first choice for us. Uterine artery ligation was conducted on one patient.

7. The lower transverse incision at the uterine was sutured in one layer in the common way, or two layers if necessary. As we can see in Figure D, this area was still permeated with tortuous vessels. In some cases, if suturing the second layer was considered dangerous, we preferred monolayer suturing. 
Short-term prognosis was analyzed. Short-term complications included bladder injury, fever, late puerperal hemorrhage, pyometra, peritonitis, uterine necrosis, sepsis and perioperative infection, hematoma around the puncture location, false aneurysm, arteriovenous fistula and thrombus. All 104 patients who had an abdominal aorta artery balloon were ordered to take an echo examination after the surgery to check whether there was thrombus in the vessels (artery from common iliac artery to dorsalis pedis artery and vein from dorsalis pedis vein to common iliac vein) of the pelvis and extremities.

Long-term complications were followed up. Short-term follow-up was conducted for all the patients at outpatient department 42 days after PAMRR. Long-term follow-up was conducted three months, six months, one year, and two years after the surgery. Breast feeding situation, menstrual quantity, menstrual period, chronic pelvic pain, intrauterine adhesion and lower extremity discomfort were followed-up. One case who was pregnant after PAMRR was reported particularly.

\section{Results}

All 142 patients suffered from complete placenta previa. The characteristics of the population are listed in Table 1. The perioperative data of patients are listed in Table 2. 
Table 1

Demographic characteristics of the patients with placenta percreta treated by PAMRR

\begin{tabular}{|lll|}
\hline Variables & No. of patients & $\%$ \\
\hline Age & 42 & \\
\hline$<30$ years & 52 & 29.5 \\
$30-34$ years & 48 & 36.7 \\
$\geq 35$ years & & 33.8 \\
\hline Gravidity & 52 & \\
\hline$\leq 3$ & 73 & 36.7 \\
\hline $4-5$ & 17 & 51.4 \\
$\geq 6$ & & 11.9 \\
\hline Number of previous C-Sections & 82 & 57.8 \\
1 & 51 & 35.9 \\
2 & 9 & 6.3 \\
\hline$>2$ & & \\
\hline Gestation age & 28 & 19.7 \\
\hline$<35$ weeks & 38 & 56.3 \\
\hline $35-37$ weeks & 34 & 24 \\
\hline$>37$ weeks & & \\
\hline Hospital stay & & \\
\hline $4-10$ days & & \\
\hline$>10$ days & & \\
\hline
\end{tabular}


Table 2

\begin{tabular}{|c|c|c|}
\hline Variables & No. of women & $\%$ \\
\hline \multicolumn{3}{|c|}{ Estimated Blood loss (ml) } \\
\hline$<1000$ & 58 & 40.9 \\
\hline $1000-2000$ & 57 & 40.1 \\
\hline$\geq 2000$ & 27 & 19 \\
\hline \multicolumn{3}{|c|}{ The amount of packed red blood cells transfused } \\
\hline 0 unit & 50 & 35.2 \\
\hline$\leq 6$ unit & 72 & 50.7 \\
\hline$>6$ unit & 20 & 14.1 \\
\hline \multicolumn{3}{|c|}{ The amount of auto blood transfused } \\
\hline none & 89 & 62.7 \\
\hline$<500 \mathrm{ml}$ & 32 & 22.5 \\
\hline$>500 \mathrm{ml}$ & 21 & 14.8 \\
\hline \multicolumn{3}{|c|}{ The amount of fresh-frozen plasma transfused } \\
\hline $0 \mathrm{ml}$ & 85 & 59.9 \\
\hline$<1000 \mathrm{ml}$ & 50 & 35.2 \\
\hline$\geq 1000$ & 7 & 4.9 \\
\hline \multicolumn{3}{|l|}{ Operation time } \\
\hline$<100 \min$ & 71 & 50 \\
\hline $100-150 \mathrm{~min}$ & 62 & 43.7 \\
\hline$>150$ min & 9 & 6.3 \\
\hline
\end{tabular}

The procedure was conducted under tourniquet in only 33 cases, and under prophylactic abdominal aorta balloon occlusion in only 29 cases. In addition, 75 cases were treated under both means, while 5 cases were treated by neither of the methods. The postoperative results of two patients failed to meet our satisfaction. We needed to embolize the uterus artery and the iliac artery to stop the bleeding. Intrauterine packing was carried out before embolism on one of the patients. But all the uteruses were preserved successfully. Bladder injury occurred in 3 patients. The muscle layer of the bladder was invaded by placenta in all the 3 patients. The injury happened when pushing down the bladder and separating the reflection between bladder and uterus. One case who used a urine tube for 18 days in order to improve bladder healing was confirmed with a urinary fungal infection. The urine tube was carried for 3 and 14 
days respectively on the other two patients. Tube ligation was performed on 111 patients considering their opinions and the risk of next pregnancy while 31 patients refused peripartum sterilization. One patient was sent to the intensive care unit (ICU) because of hemorrhagic shock.

\section{Follow-up}

The follow-up data of the population are listed in Table 3. 
Table 3

Follow-up Data of the patients with placenta percreta treated by PAMRR

\begin{tabular}{|c|c|c|}
\hline Variables & No. of patients & $\%$ \\
\hline Fever & 10 & 7.0 \\
\hline Hematoma around the puncture location & 3 & 2.1 \\
\hline Thrombus & 4 & 2.8 \\
\hline \multicolumn{3}{|l|}{ Long-term follow-up } \\
\hline Succeeded & 127 & 89.4 \\
\hline Lost & 15 & 10.6 \\
\hline \multicolumn{3}{|l|}{ Time after the procedure } \\
\hline more than 2 years & 49 & 34.5 \\
\hline more than 1 year & 113 & 79.6 \\
\hline more than six months & 124 & 87.3 \\
\hline \multicolumn{3}{|l|}{ Breast feeding situation } \\
\hline Artificial feeding & 14 & 11.0 \\
\hline Breast feeding & 113 & 89.0 \\
\hline \multicolumn{3}{|l|}{ Menstruation restored } \\
\hline Yes & 121 & 95.3 \\
\hline No & 6 & 4.7 \\
\hline \multicolumn{3}{|l|}{ Time of menstruation resumed } \\
\hline$<5$ months & 67 & 52.8 \\
\hline $5-10$ months & 42 & 33.1 \\
\hline$>10$ months & 18 & 14.1. \\
\hline \multicolumn{3}{|l|}{ Menstrual quantity } \\
\hline Increase & 5 & 4.1 \\
\hline Same & 104 & 86.0 \\
\hline Decrease & 12 & 9.9 \\
\hline \multicolumn{3}{|l|}{ Menstrual period (days) } \\
\hline Longer & 11 & 9.1 \\
\hline
\end{tabular}




\begin{tabular}{|lll|}
\hline Variables & No. of patients & $\%$ \\
\hline Same & 106 & 87.6 \\
\hline Shorter & 4 & 3.3 \\
\hline Suspicious intrauterine adhesion & 1 & 0.8 \\
\hline Light chronic pain of abdomen & 9 & 7.1 \\
\hline Lower extremity discomfort & 6 & 4.7 \\
\hline
\end{tabular}

\section{Short-term Complications}

10 patients got a brief low and moderate puerperal fever after surgery $\left(37.3^{\circ} \mathrm{C}\right.$

Three cases got hematoma around the puncture location, one of which was small and disappeared before discharge. The hematoma was larger than $10 \mathrm{~cm}$ in the other 2 patients. Full recovery was confirmed in one of the two cases. She had a pretty low platelet count. Extensive subcutaneous ecchymosis was found at the same time. But the other patient had schizophrenia and was lost to followup. Compression on the puncture location could not be carried out effectively because of schizophrenia in this patient, which was probably the main reason for the hematoma. No pyometra, peritonitis, uterine necrosis, sepsis or perioperative infection of any other system occurred. No late puerperal hemorrhage, false aneurysm, or arteriovenous fistula occurred.

Thrombus was found in 4 cases. All the thrombi were found around the puncture location. One of the patients got artery and venous thrombus, including the common femoral artery, external iliac artery and common iliac vein which may be have been caused by compression in case of hemorrhage. The specific description of the situation and treatment and long-term outcome is listed in Table 4. All the four patients were transferred to the Department of Interventional Radiology and treatment was conducted by the interventional doctor of our team. 
Table 4

The specific description of the thrombus and treatment and long-time outcome

\begin{tabular}{|lllll|}
\hline Case & Location of the thrombus & Treatment & $\begin{array}{l}\text { Time after the } \\
\text { surgery } \\
\text { (months) }\end{array}$ & $\begin{array}{c}\text { Long-term } \\
\text { outcome }\end{array}$ \\
\hline 1 & $\begin{array}{l}\text { External Iliac Artery and } \\
\text { common femoral artery and } \\
\text { common iliac vein }\end{array}$ & $\begin{array}{l}\text { warfarin and } \\
\text { thrombolysis by } \\
\text { urokinase }\end{array}$ & 32 & normal \\
\hline 2 & $\begin{array}{l}\text { External Iliac Artery and } \\
\text { common femoral artery }\end{array}$ & $\begin{array}{l}\text { arteriotomy with } \\
\text { embolectomy }\end{array}$ & 25 & normal \\
\hline 3 & $\begin{array}{l}\text { External Iliac Artery and } \\
\text { common femoral artery }\end{array}$ & $\begin{array}{l}\text { arteriotomy with } \\
\text { embolectomy }\end{array}$ & 31 & normal \\
\hline 4 & $\begin{array}{l}\text { Common femoral artery } \\
\text { catheter-directed } \\
\text { thrombolysis by } \\
\text { urokinase }\end{array}$ & 30 & $\begin{array}{l}\text { numbness } \\
\text { in right } \\
\text { knee }\end{array}$ \\
\hline
\end{tabular}

\section{Long-term Complications}

Up to March 31, 2020, 15 cases out of the 142 patients were lost to follow-up while the other 127 cases were followed-up successfully by phone for 3 to 38 months after the surgery by the same doctor, Huidan Zhao.

Menstrual quantity decreased in 12 patients after the surgery. And it reduced more than half in 5 out of the 12 cases, but ultrasonic examination of all the 12 patients was normal. Menstrual period became shorter than before in 4 cases, at the same time the menstrual quantity decreased in all 4 patients. Prolongation of menstrual cycle happened in 11 cases and ranged from 3 to 12 days. Scar diverticulum was diagnosed in 3 cases by transvaginal ultrasound, while 8 patients were normal.

There were 9 patients who got other uncomfortable symptoms in the lower abdomen, including 3 cases during intercourse and 1 case before urination.

Suspicious intrauterine adhesion was found in one patient. The operation was conducted under tourniquet and prophylactic abdominal aorta balloon occlusion two year previously. Three months later her menstruation restored. But irregular vaginal bleeding happened every month after menstruation and lasted for 8-9 days, accompanied by chronic pain in the right lower abdomen. Adhesion was suggested by transvaginal ultrasound. Hysteroscopy was not performed because of personal reasons.

Lower extremity discomfort was found in 6 patients. Abdominal aorta balloon was used on all of them. The main symptoms were slight sense recession, numbness and soreness in the leg, knee or ankle, which arose from maintaining the same posture for a long time, staying in the cold weather or doing exercise for a long time. One patient was diagnosed with thrombosis at the puncture location. Vessel radiography and 
ultrasonic examination were normal after treatment. The remaining 5 cases were confirmed with normal ultrasound performance in the routine inspection two days after the surgery.

A 29-year-old woman with placenta percreta on whom we performed the surgery on November 2017 was pregnant and delivered a baby on April 2020. We emphasized the risk of next pregnancy, but she declined sterilization because this was her first baby of her new family after divorce. The placenta was located at the posterior wall far away from the internal os of the cervix. An elective caesarean section with sterilization was performed at 38 weeks' gestation successfully.

\section{Discussion}

As numbers of patients with placenta previa accreta increase, many uterus compression sutures have been reported in succession in order to minimize the bleeding of uterus, like distinguished B-Lynch surgical technique, multiple square suture, parallel vertical compression suture, Triple-P procedure and so on $[3-5,7]$. Every compression suture has their corresponding indications and advantages, with the main performance of uterine atony and placenta previa. But our method aimed at extensive placenta percreta. Prophylactic devascularization achieved by aortic artery balloon occlusion and/or tourniquet before dealing with the placenta can reduce the risk of intra operative blood loss and prevent the occurrence of severe postpartum hemorrhage [10-18]. First, the eroded defective anterior lower uterus segment and bulk of the placenta without detachment was removed together, which can reduce the homeostasis area and shorten the homeostasis time, consequently reducing the bleeding volume. Second, once partial anterior uterus myometrium has been resected, the internal os of the cervix is easier to expose, greatly facilitating next surgical homeostasis suture applied to this area. Third, only the eroded unfunctional uterus tissue was excised and the normal myometrium was preserved as much as possible. After reconstruction, the incision was the same as in a traditional C-section. Compared with other operations, this method has the advantage of less trauma.

The "Triple-P procedure" put forward by British researchers Chandraharan E at al involves perioperative placental localization, pelvic devascularization, placental nonseparation with myometrial excision and reconstruction of the uterine wall, which can effectively preserve the uterus [4]. The procedure we reported is similar to the Triple-P procedure. But there are some differences, too. First, in the Triple-P procedure, the occlusion balloon catheters are placed in the bilateral internal iliac artery, and are required to puncture both sides of the femoral artery. Whereas in our study, the occlusion balloon catheter was placed in the abdominal aorta artery. It only needed to puncture one side of femoral artery. This process meant a much lower risk of thrombosis and promised more effective devascularization. Second, for some patients, occlusion balloon and tourniquet were used simultaneously, which can achieve maximum occlusion of the blood supply to the uterus. Third, we pushed down the bladder, exposed the lower segment of uterus and excised as mush placenta with the eroded unfunctional uterus tissue as possible; the Triple-P procedure does not mention the bladder. Forth, a single continuous suture was carried out on the lower lip of the uterine incision which was one part of placenta percreta area and was full of tortuous vessels. This step can reduce the bleeding dramatically. 
There are very limited data about the long-term complications associated with the use of uterus compression techniques. For previous sutures, the long-term complications cannot be identified very easily due to insufficient cases and unsustainable follow-up. We have implemented this procedure for about six years. Changes of menstrual and lower extremity discomfort cannot be ignored.

We made some different discoveries from previous reports. Lower limb complaints were found in quite a few cases. 5 out of 6 cases experienced discomfort on the right side, while 1 case on the both sides. The reason why lower limb complaints happened to patients with normal echo performance was unclear. We inferred two possible reasons. First, it may be relative to ischemic injury to femoral nerve. Second, although there was no thrombosis found in the big artery through vessel radiography and echo examination, it was unclear whether there was small thrombosis in the peripheral artery.

With the improvement of medical technology, more and more interventional procedures appearing and they are being applied to obstetric disease, including temporal balloon occlusion of internal iliac artery [19], common iliac artery $[20,21]$ or abdominal aorta artery $[10,13,14]$ and embolization of uterine artery or internal iliac artery. We all focus on the advantages of these procedures. But most of us have not paid enough attention to the disadvantages. Although there were some reports about the complications of interventional radiological procedures, including thrombus formation, pseudoaneurysm, arterial rupture and so on. But most of them were case report and the long-term outcomes have rarely been recorded and reported [22-24]. From our study, we can conclude that even if the imaging examination of the vessels of pelvis and lower extremities is absolutely normal, patients after the interventional surgery can have uncomfortable symptoms for a long time. Hematoma and thrombosis are not rare actually, which is different from previous researches. So, we must take the indications of interventional measures seriously and should not overuse the double-edged sword easily.

\section{Conclusions}

In summary, combined with tourniquet and/or prophylactic abdominal aorta balloon occlusion, Partial Anterior Myometrial Resection and Reconstruction (PAMRR) is highly effective in reducing the intraoperative blood loss and hysterectomy in patients with placenta percreta. It is a safe and effective surgical alternative to peripartum hysterectomy. But we should also pay attention to the complications of abdominal aorta balloon occlusion.

\section{Abbreviations}

PAS: Placenta accreta spectrum; EBL: Estimated blood loss; DIC: Disseminated intravascular coagulation; MOF: Multi-organ failure; PPH: Postpartum hemorrhage; PAMRR: Partial Anterior Myometrial Resection and Reconstruction; MRI: Magnetic Resonance Imaging; DSA: Digital subtraction angiography; ICU: Intensive care unit.

\section{Declarations}


Our manuscripts contain the following sections: ethics approval and consent to participate, consent for publication, availability of data and materials, competing interests, funding, authors' contributions, acknowledgements and authors' information.

\section{Acknowledgements}

Not applicable.

\section{Contribution to authorship}

Xianlan Zhao (corresponding author) designed the surgical technique and guarantees all aspects of the reliability. Huidan Zhao carried out the data collection, performed statistical analyses and wrote this article. Xianlan Zhao, Huidan Zhao, Ya Tao, Zhuan Liu and Cai Liu were the principal surgeons who performed or participated in these operations. Chen Chen made the illustration of Figure and revised the manuscript. All authors approved the final version of the article.

\section{Funding}

No specific funding obtained.

\section{Availability of data and materials}

We would like to share the database upon individual request.

\section{Ethics approval and consent to participate}

All procedures performed in studies were in accordance with the ethical standards of the institutional research committee (Ethics Committee of the First Affiliated Hospital of Zhengzhou University). All the subjects were informed of the study purpose. Written informed consent was obtained for each patient.

\section{Consent for publication}

The patient who was pregnant and delivered a baby after the surgery described in the manuscript granted written informed consent to publish.

\section{Competing interests}

All authors declare no conflict of interest.

\section{Author details}

1 Department of Obstetrics, the First Affiliated Hospital of Zhengzhou University, Zhengzhou, China

2 Obstetric Emergency and Critical Care Medicine of Henan Province, Zhengzhou, China 


\section{References}

1. Jauniaux E, Ayres-de-Campos D, Diagnosis FPA. Management Expert Consensus P. FIGO consensus guidelines on placenta accreta spectrum disorders: Introduction. Int J Gynaecol Obstet. 2018;140(3):261-4.

2. Jauniaux E, Alfirevic Z, Bhide AG, Belfort MA, Burton GJ, Collins SL, et al. Placenta Praevia and Placenta Accreta: Diagnosis and Management: Green-top Guideline No. 27a. BJOG. 2019;126(1):e148.

3. C BL, Coker A, Lawal AH, Abu J, Cowen MJ. The B-Lynch surgical technique for the control of massive postpartum haemorrhage: an alternative to hysterectomy? Five cases reported. $\mathrm{Br} \mathrm{J}$ Obstet Gynaecol. 1997;104(3):372-5.

4. Chandraharan E, Rao S, Belli AM, Arulkumaran S. The Triple-P procedure as a conservative surgical alternative to peripartum hysterectomy for placenta percreta. Int J Gynaecol Obstet. 2012;117(2):191-4.

5. GT L, GR L, XF L, BP W. Funnel compression suture: a conservative procedure to control postpartum bleeding from the lower uterine segment. BJOG: an international journal of obstetrics gynaecology. 2016;123(8):1380-5.

6. JC S, KL L, JH JK, MW Y, CU L. Y. 'Nausicaa' compression suture: a simple and effective alternative to hysterectomy in placenta accreta spectrum and other causes of severe postpartum haemorrhage. BJOG: an international journal of obstetrics gynaecology. 2019;126(3):412-7.

7. YM H, CP C, HS C. TH S. Parallel vertical compression sutures: a technique to control bleeding from placenta praevia or accreta during caesarean section. BJOG: an international journal of obstetrics gynaecology. 2005;112(10):1420-3.

8. JH C, HS J. CN L. Hemostatic suturing technique for uterine bleeding during cesarean delivery. Obstetrics gynecology. 2000;96(1):129-31.

9. RG H. S A, PJ S. Uterine compression sutures: surgical management of postpartum hemorrhage. Obstetrics gynecology. 2002;99(3):502-6.

10. Wei X, Zhang J, Chu Q, Du Y, Xing N, Xu X, et al. Prophylactic abdominal aorta balloon occlusion during caesarean section: a retrospective case series. Int J Obstet Anesth. 2016;27:3-8.

11. Xie L, Wang Y, Luo FY, Man YC, Zhao XL. Prophylactic use of an infrarenal abdominal aorta balloon catheter in pregnancies complicated by placenta accreta. J Obstet Gynaecol. 2017;37(5):557-61.

12. Duan XH, Wang YL, Han XW, Chen ZM, Chu QJ, Wang L, et al. Caesarean section combined with temporary aortic balloon occlusion followed by uterine artery embolisation for the management of placenta accreta. Clin Radiol. 2015;70(9):932-7.

13. Wu Q, Liu Z, Zhao X, Liu C, Wang Y, Chu Q, et al. Outcome of Pregnancies After Balloon Occlusion of the Infrarenal Abdominal Aorta During Caesarean in 230 Patients With Placenta Praevia Accreta. Cardiovasc Intervent Radiol. 2016;39(11):1573-9. 
14. Wang YL, Duan XH, Han XW, Wang L, Zhao XL, Chen ZM, et al. Comparison of temporary abdominal aortic occlusion with internal iliac artery occlusion for patients with placenta accreta - a nonrandomised prospective study. Vasa. 2017;46(1):53-7.

15. Zeng C, Yang M, Ding Y, Yu L, Deng W, Hu Y, et al. Preoperative infrarenal abdominal aorta balloon catheter occlusion combined with Bakri tamponade reduced maternal morbidity of placenta increta/percreta. Med (Baltim). 2017;96(38):e8114.

16. Chen L, Wang X, Wang H, Li Q, Shan N, Qi H. Clinical evaluation of prophylactic abdominal aortic balloon occlusion in patients with placenta accreta: a systematic review and meta-analysis. BMC Pregnancy Childbirth. 2019;19(1):30.

17. Ikeda T, Sameshima H, Kawaguchi H, Yamauchi N, Ikenoue T. Tourniquet technique prevents profuse blood loss in placenta accreta cesarean section. J Obstet Gynaecol Res. 2005;31(1):27-31.

18. Meng JL, Gong WY, Wang S, Ni XJ, Zuo CT, Gu YZ. Two-tourniquet sequential blocking as a simple intervention for hemorrhage during cesarean delivery for placenta previa accreta. Int $J$ Gynaecol Obstet. 2017;138(3):361-2.

19. Carnevale FC, Kondo MM, de Oliveira Sousa W Jr, Santos AB, da Leal Filho M, Moreira JM AM, et al. Perioperative temporary occlusion of the internal iliac arteries as prophylaxis in cesarean section at risk of hemorrhage in placenta accreta. Cardiovasc Intervent Radiol. 2011;34(4):758 - 64.

20. Minas V, Gul N, Shaw E, Mwenenchanya S. Prophylactic balloon occlusion of the common iliac arteries for the management of suspected placenta accreta/percreta: conclusions from a short case series. Arch Gynecol Obstet. 2015;291(2):461-5.

21. Shih JC, Liu KL, Shyu MK. Temporary balloon occlusion of the common iliac artery: new approach to bleeding control during cesarean hysterectomy for placenta percreta. Am J Obstet Gynecol. 2005;193(5):1756-8.

22. Chouliaras S, Hickling DJ, Tuck JS. Thromboembolism of the leg following prophylactic balloon occlusion of the uterine arteries. BJOG. 2009;116(9):1278-9.

23. Gagnon J, Boucher L, Kaufman I, Brown R, Moore A. Iliac artery rupture related to balloon insertion for placenta accreta causing maternal hemorrhage and neonatal compromise. Can J Anaesth. 2013;60(12):1212-7.

24. Bishop S, Butler K, Monaghan S, Chan K, Murphy G, Edozien L. Multiple complications following the use of prophylactic internal iliac artery balloon catheterisation in a patient with placenta percreta. Int J Obstet Anesth. 2011;20(1):70-3.

\section{Figures}



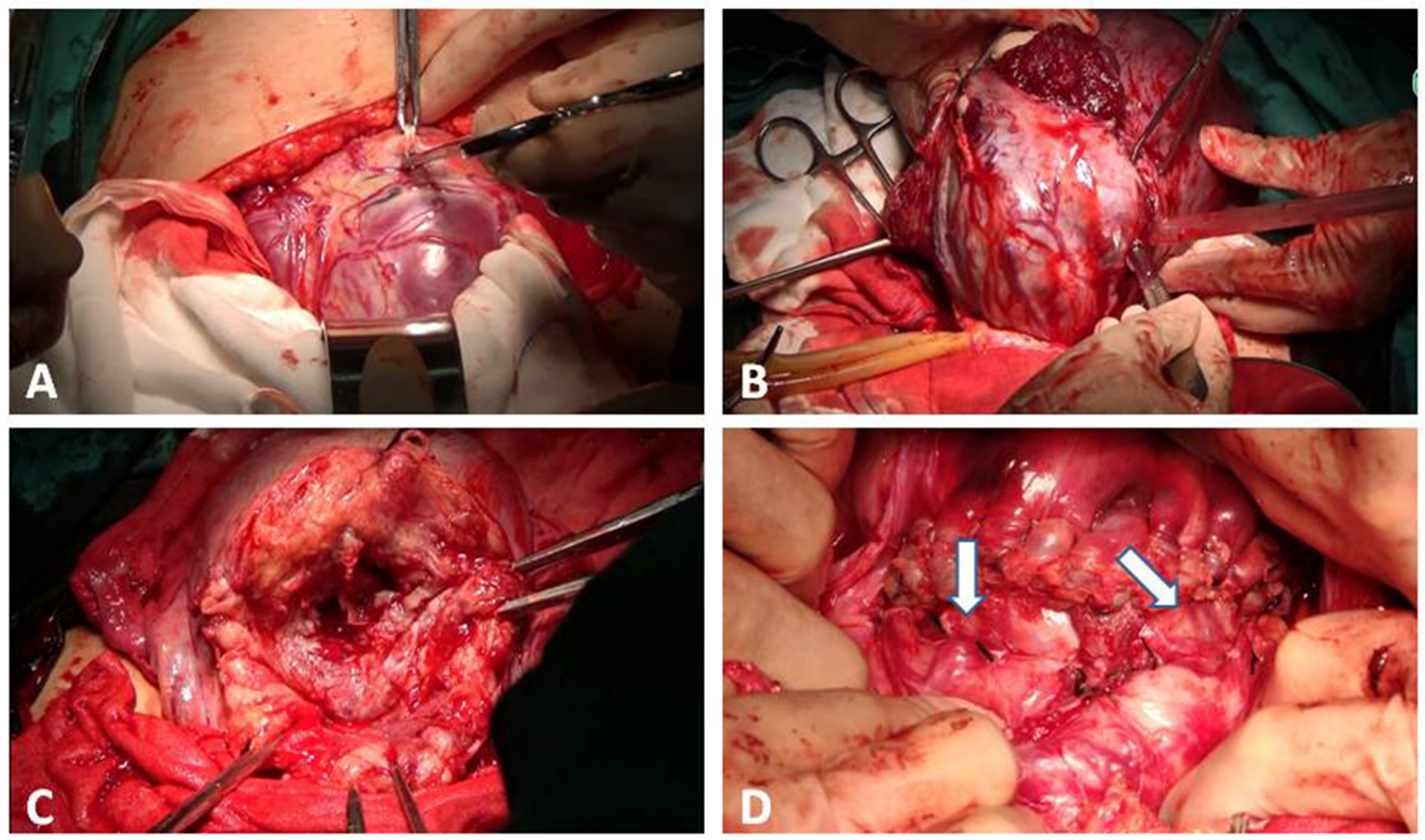

\section{Figure 1}

A. The uterine incision was over the upper border of the placenta. B. A tourniquet was put as low as possible at the lower segment of the uterus. The bladder was pushed down and the anterior myometrium was partially resected with the placenta unseparated. C. A single continuous suture was made along the lower lip of the uterine incision. D. The lower transverse incision at the uterine was sutured in one layer. This area was still permeated with tortuous vessels (the arrow). 